\title{
Evaluación de Ventanas Fotovoltaicas con Concentradores Solares Luminiscentes para Edificios Cero-energía en Santiago de Chile
}

\section{Evaluation of Photovoltaic Windows with Luminescent Solar Concentrators for Zero-energy Buildings in Santiago de Chile}

\author{
J. Alarcón-Castro $^{(*)}, \underline{\text { R. García-Alvarado }}{ }^{(*)}$, P. Sanchéz-Friera ${ }^{(* *)}$, A. Zapico-Ania ${ }^{(* *)}$
}

\section{RESUMEN}

Los vidrios con concentradores solares luminiscentes (LSC) permiten plantear ventanas con recolección fotovoltaica perimetral, como elementos integrados en la edificación (BIPV). Este trabajo evalúa su aplicación en edificios de oficina en Santiago de Chile, para aportar en metas de cero-energía, revisando primeramente los planes energéticos para la edificación en Europa y Chile, los conceptos de cero-energía en edificios y definiendo un modelo de estudio en Santiago de Chile. Luego se simula el rendimiento y generación energética con distintas propiedades constructivas, disposición y eficiencia de las ventanas. El análisis sugiere que esta tecnología, dispuesta en extensiones amplias hacia la fachada soleada, puede otorgar hasta un 40\% del consumo para edificios longitudinales con envolvente y equipamientos eficientes, además de contribuir al sombreamiento. Se demuestra por tanto que, regulando la edificación y conformando ventanas extensas adecuadamente dispuestas, esta tecnología puede contribuir significativamente a alcanzar las metas de edificios cero-energía.

Palabras clave: Concentradores Solares Luminiscentes, Ventanas Inteligentes, Elementos Fotovoltaicos Integrados en la Edificación, Edificios Cero-Energía, Santiago.

\section{ABSTRACT}

Glasses with luminescent solar concentrators (LSC) allow to develop windows with perimeter photovoltaic collection, as building-integrated photovoltaics (BIPV). This work assesses its application in office buildings in Santiago de Chile, to contribute in zero-energy goals, reviewing energy plans for building in Europe and Chile, zero-energy concepts in buildings and defining a study model in Santiago de Chile. Then the performance and energy generation are simulated with different constructive properties, layout and efficiency of the windows. The analysis suggests that this technology, when used in wide areas over the sunniest façades, can provide up to $40 \%$ of building consumption, for longitudinal buildings with energy-efficient envelope and equipment, in addition to contributing to shading. It is thus demonstrated that, by regulating the building and forming large windows adequately arranged, this technology can contribute significantly to reach zero-energy buildings goals.

Keywords: Luminescent Solar Concentrators, Smart Windows, Building-Integrated Photovoltaics, Zero-energy Buildings, Santiago.

(*) Universidad de Bio-Bio (Chile).

(**) Fundación Prodintec (España).

Persona de contacto/Corresponding author: rgarcia@ubiobio.cl (R. García-Alvarado)

ORCID: http://orcid.org/oooo-0oo2-4324-4369 (J. Alarcón-Castro); http://orcid.org/oooo-0oo3-2216-2388

(R. García-Alvarado); http://orcid.org/oooo-0oo2-9321-4248 (P. Sanchéz-Friera);

http://orcid.org/oooo-0001-5431-6789 (A. Zapico-Aniaco)

Cómo citar este artículo/Citation: J. Alarcón-Castro, R. García-Alvarado, P. Sanchéz-Friera, A. Zapico-Ania. (2018). Evaluación de Ventanas Fotovoltaicas con Concentradores Solares Luminiscentes para Edificios Cero-energía en Santiago de Chile. Informes de la Construcción, 70(550): e255. https://doi.org/10.3989/id.58081

Copyright: (c) 2018 CSIC. Este es un artículo de acceso abierto distribuido bajo los términos de la licencia de uso y distribución Creative Commons Reconocimiento 4.o Internacional (CC BY 4.0). 


\section{INTRODUCCIÓN}

El desarrollo de vidrios con concentradores solares luminiscentes (LSC: luminiscent solar concentrators) es una tecnología prometedora para elementos fotovoltaicos integrados en los edificios (BIPV: building-integrated photovoltaic), que pueden proveer iluminación natural y además, generar energía limpia y gratuita, contribuyendo a la reducción de los consumos energéticos y disminución del impacto ambiental. La tecnología LSC consiste en adherir una capa translúcida en el vidrio, con partículas activas que pueden capturar gran parte del espectro de radiación solar incidente, canalizada mediante mallas poliméricas al perímetro en que se colocan colectores fotovoltaicos $(1,2$, 3). Por lo que pueden aplicarse en paramentos vidriados, como ventanas, cubiertas o fachadas acristaladas que pueden mantener su función regular y producir energía. Sin embargo, no se han difundido todavía productos industrializados con esta tecnología, ni se ha evaluado su aplicación en los edificios (4). El proyecto WINNER de la Convocatoria ERANET-LAC 2014, en realización por la Fundación Prodintec de España, Centro de Nanotecnología y Materiales Inteligentes (CENTI) de Portugal, Universidad Nacional Evangélica (UNEV) de República Dominicana y la Universidad del Bío-Bío (UBB) de Chile, pretende desarrollar un sistema de ventanas fotovoltaicas con concentradores solares luminiscentes para aportar a edificios cero-energía. Este trabajo realiza una evaluación de su aplicación en un caso de estudio de edificio de oficinas en Santiago de Chile, para determinar las características más apropiadas y orientar el diseño de la ventana. Comenzando por una revisión de los planes energéticos para la edificación en Europa y Chile, y la definición de un modelo de estudio en Santiago de Chile, a continuación se evalúa por simulaciones dinámicas la eficiencia y generación energética con distintas propiedades constructivas, disposición y desempeño de las ventanas.

\subsection{Edificios Cero-Energía}

El concepto de edificios cero-energía ha emergido los últimos años, a partir de la tendencia de desarrollo sostenible, como una meta de reducción de consumos energéticos, combinando acciones de eficiencia energética e integración de sistemas de generación renovable. Según un estudio del Departamento de Energía de los Estados Unidos (5), el concepto de edificio cero-energía (ZEB: Zero-energy Building) puede tener cuatro acepciones;

a) De sitio; combinando todas las alimentaciones y requerimientos de energía primaria en el sitio del edificio.

b) De fuentes; que relaciona las fuentes de origen de las energías, evaluando su conversión.

c) De costos; que evalúa la compensación energética según el valor económico de las distintas energías urbanas.

d) De emisiones; que evalúa la compensación energética de acuerdo a la emisión de gases invernadero de cada tipo de combustible y producción.

La primera acepción es más limitada, pero más fácil de evaluar, por lo que se ha popularizado. Recientemente esta misma entidad planteó una definición de edificio cero-energía basada en las fuentes de energía, considerando que el balance anual entre la demanda regular sea menor o igual que la energía renovable exportada desde el sitio (6).
La Comunidad Europea, a su vez, planteó la definición de Edificios de Casi-Cero Energía (NZEB: Near Zero Energy Building), a través de la Directiva 2010/31, comprometiendo para el 2020 su adopción en todos los edificios nuevos y existentes de propiedad gubernamental (7). Definiendo que deben ser edificios de muy alto rendimiento con bajos requerimientos de energía, asumida en gran medida de fuentes renovables, lo que se calcula según energía primaria (en kilovatio-hora por m2 de superficie ocupada al año), de los servicios regulares (8). La Federación Europea de Organizaciones Profesionales de Climatización (REHVA) ha intentado normalizar esta definición, expresando los flujos energéticos involucrados en el edificio (9), y para analizar el balance energético se ha popularizado una gráfica de valores de demanda frente a generación en $\mathrm{kWh} / \mathrm{m} 2-\mathrm{y}$ (10). Actualmente se dispone de la norma ISO EN 52000-1, con un apéndice de definición NZEB. Aunque existen controversias en el establecimiento de métricas y alcances del concepto $(11,12)$, como también si se abordan elementos externos al edificio o conjuntos de edificios de la misma propiedad, gestión o localización (13), sobre el periodo de análisis, ya sea diario, mensual, anual o del ciclo de vida completo (12), y la relación con características de diseño del edificio (14).

En Reino Unido se ha difundido el concepto de edificios Cero-Carbón (ZCB: Zero-Carbon Building), que contempla medir demanda energética $(\mathrm{kWh} / \mathrm{m} 2-\mathrm{y})$, consumo de $\mathrm{CO} 2$ (en $\mathrm{kg} / \mathrm{m} 2-\mathrm{y})$ y costes de operación y ejecución. Otra acepción frecuente es Edificios Cero-Energía en Red (NetZEB), promovida por instituciones norteamericanas (15) que considera una reducción de las necesidades energéticas compensadas con alimentación renovable vinculada a redes urbanas.

En Chile, desde los años 80 se han realizado estudios y sugerencias normativas sobre requerimientos energéticos en edificación, y el año 2000 se implementó, por primera vez en Latinoamérica, una legislación constructiva destinada a mejorar el rendimiento térmico de viviendas, además de iniciar programas de promoción a la eficiencia energética. Chile ha sido pionero dentro del continente sobre materias energéticas de edificación debido a la carencia de fuentes combustibles propias y poseer climas más templados y variables, además de un fuerte desarrollo de la construcción. En los últimos años se han implementado además certificaciones de vivienda y edificios sostenibles, pero de manera voluntaria y sin metas de rendimiento. En el año 2012 se planteó un manual de gestión de energía en edificios públicos (16), y posteriormente una estrategia nacional de construcción sostenible (17) que propone una reducción del $12 \%$ del consumo al 2020, y generar un $10 \%$ con renovables. Esto se refuerza con Planes de Descontaminación Atmosférica (18) que otorgan fondos de reacondicionamiento térmico de viviendas por ciudades, y programas de fomento estatales (19). Además la política energética nacional (20) plantea implementar en todas las edificaciones, condiciones constructivas eficientes, mejora de confort y gestión inteligente, así como un 70\% de renovables en la matriz nacional de generación. Estos planteamientos, con metas más reducidas y orientaciones más prescriptivas o administrativas, provienen de un nivel de desarrollo más limitado, en la cual la aplicación de restricciones produce usualmente efectos de rebote (aumentar el consumo por carencias de confort), aunque igualmente son convergentes en una eficiencia energética e integración de renovables. Por ende, un enfoque de 
edificio de cero-energía es pertinente analizar en este contexto, para contribuir a políticas de construcción sostenible señeras en el continente.

Para este trabajo consideramos que un edificio cero-energía debe poseer una demanda energética menor que la usual para edificios con actividades y localización similar, a través de mejoras constructivas o de equipamiento, y que su balance anual incluya provisión de fuentes renovables generadas en el propio edificio o cercanamente. Las condiciones de emisión, relación con redes urbanas y costes dependen de legislaciones o capacidades más circunstanciales, por tanto para este análisis se omiten, así como los sistemas de gestión, concentrándose en determinar energía demandada o requerida del edificio. Así mismo, se considera una equivalencia horaria para la provisión/demanda energética del propio edificio, excluyendo costes de almacenamiento o tarifación por redes, $\mathrm{y}$ desequilibrios entre fuentes urbanas.

\subsection{Estado del Arte}

La aplicación de ventanas fotovoltaicas con concentradores solares luminiscentes debe cumplir funciones de iluminación natural, vistas y/o ventilación, adicionando propiedades de generación energética solar (21), a diferencia de los paneles que se sobreponen adicionalmente al edificio, o se despliegan en áreas abiertas, que están dedicados exclusivamente a recolección. En varios edificios alrededor del mundo se han instalado generadores fotovoltaicos integrados en cubiertas, muros, balcones o áreas vidriadas (con celdas insertas), pero aún no se han generalizado tecnologías o evaluaciones al respecto. Una revisión de una treintena de edificios que han implementado elementos fotovoltaicos, algunos sobrepuestos y otros integrados, presenta una relevante cantidad (cerca de la mitad) que logra cubrir la totalidad de la demanda energética (22).

Algunos estudios sobre sobre elementos constructivos fotovoltaicos para lograr edificios casi cero-energía (NZEB), han determinado la relevancia de la tipología del edificio y su localización para alcanzar esta meta (23). Es decir, su implementación adecuada es dependiente de la configuración, ocupación y situación del edificio. Otro trabajo ha sugerido la instalación en oficinas y centros comerciales de latitudes medias con buena radiación solar (24), aunque sin caracterizar su rendimiento. El proyecto SWING de la empresa italiana ENEL y el Politécnico de Milano ha experimentado ventanas con cristales con concentradores solares luminiscentes, pero como unidades autónomas, sin considerar su participación en el comportamiento total del edificio (25), determinando aportes relevantes, pero advirtiendo limitaciones por el rango de color utilizado.

\section{MATERIALES Y MÉTODOS}

\subsection{Edificios de Oficina en Santiago de Chile}

Santiago de Chile es una de las capitales más pujantes de América Latina, con cerca de 6.000.00o de habitantes y aprox. 400.000.00o de m2 construidos, de los cuales un tercio corresponde a edificios para servicios, industria, comercio y finanzas, y cada año se construyen más de 1.500.000 m2 (26). Éstos generan cerca de un tercio del consumo energético total, alrededor de 20.000 GWh anuales, estimando unos $150 \mathrm{kWh} / \mathrm{m} 2-\mathrm{y}$, con un crecimiento anual del $3 \%$.
Los edificios de oficinas en Santiago de Chile se caracterizan por poseer estructuras de hormigón armado, con alturas desde uno hasta un centenar de pisos, y fachadas mixtas con cerramientos opacos y paños vidriados. Según un estudio muestral de las comunas con mayor edificación de oficinas (27), se presentan tres tipos usuales de edificios; de gran altura ( 16 pisos o más), mediana altura ( 6 a 15 pisos) y baja altura (1 a 5 pisos). Evidenciando un mayor consumo energético en los edificios más vidriados, por requerimientos de refrigeración en verano (28).

Por otro lado, Santiago de Chile posee un clima templado cálido con lluvias invernales y una larga estación seca (Tabla 1). Su área urbana está ubicada en latitud $33^{\circ} 26^{\prime} 16^{\prime \prime} \mathrm{S}$ y longitud $70^{\circ} 39^{\prime} 01^{\prime \prime} \mathrm{O}$, con una altitud media de 570 m.s.n.m.

Se han realizado variados estudios para caracterizar el rendimiento energético de los edificios de oficinas en Santiago de Chile. Una tesis de ingeniería (27), revisando 150 edificios representativos, determinó un consumo anual medio en electricidad de 95,4 kWh/m2, en gas natural 34,6 kWh/m2 y en agua potable de $1,43 \mathrm{~m} 3 / \mathrm{m} 2$. Con emisiones de $\mathrm{CO} 2$ más de $50 \mathrm{~kg} / \mathrm{m} 2$ al año, y costos anuales en energía y agua potable de 14,5 US $\$ / \mathrm{m} 2$, que corresponde al 9\% del valor de arriendo. Reconociendo un amplia dispersión de valores, por lo que el estudio discrimina según alturas de edificios. En edificios de baja altura, determina un consumo anual medio en electricidad de $86,5 \mathrm{kWh} / \mathrm{m} 2$ y $7,6 \mathrm{kWh} / \mathrm{m} 2$ mensuales, que abarca alumbrado, fuerza, computación y climatización (fundamentalmente refrigeración). Lo que coincide también con un estudio (29), que determinó para edificios de oficina en esta zona, un consumo anual medio por superficie ocupada de 86,62 kWh/m2-y, basado en registros que reconocen un predominio de los requerimientos eléctricos.

\subsection{Morfología del Caso de Estudio}

Para determinar una volumetría de edificio adecuada para la contribución energética por ventanas fotovoltaicas, se considera primero que esta tipología presenta usualmente una conformación prismática (de paralelepípedo rectangular), con amplias variaciones de dimensión, pero con una partición horizontal similar (pisos), en que se realizan las actividades. Las cuales deben mantener una proximidad a las caras verticales para iluminación natural y ventilación, limitando la magnitud horizontal (lo que se conoce como anchos de crujías). Por tanto aunque cambien las dimensiones mayores, la relación entre las superficies horizontales (que produce el consumo) y las fachadas (en que se pueden instalar ventanas) es limitada (ver Tabla 2). Cada metro longitudinal de planta posee usualmente 2,5 a $4 \mathrm{ml}$ de fachada, para 3 a $10 \mathrm{~m} 2$ de profundidad. Por tanto desde 0,3 hasta $1 \mathrm{~m} 2$ de fachada por cada metro cuadrado de piso. La proporción menor se da en los edificios mayores y más anchos, y la menor, en los más pequeños y angostos. Como la superficie de planta corresponde al área de ocupación que genera

Tabla 1. Condiciones Climatológicas de Santiago de Chile.

\begin{tabular}{|l|c|c|c|}
\hline & Enero & Julio & Anual \\
\hline Temperatura media $\left({ }^{\circ} \mathrm{C}\right)$ & 20.9 & 8.1 & 14.4 \\
\hline Horas de sol & 362.7 & 145.7 & 2897.9 \\
\hline Precipitación total $(\mathrm{mm})$ & 0.4 & 86.6 & 312.5 \\
\hline Radiación media (Kwhm2) & 7.0 & 2.4 & 1.422 \\
\hline Humedad relativa (\%) & 57 & 84 & 71 \\
\hline
\end{tabular}


Tabla 2. Morfología de Edificios de Oficinas en Santiago de Chile y Modelo de Estudio.

\begin{tabular}{|l|c|c|c|c|}
\hline & Edificios de Oficinas & Edif. Vidriados & Edif. pequeños & Modelo de Estudio \\
\hline Superficie & 200 a $20.000 \mathrm{~m} 2$ & 200 a $20.000 \mathrm{~m} 2$ & 200 a 800 & 385 \\
\hline Cantidad de Pisos & 1 a 80 & 1 a 80 & 1 a 10 & 4 \\
\hline Altura & 2,5 a $240 \mathrm{~m}$ & 2,5 a $240 \mathrm{~m}$ & 2,5 a $40 \mathrm{~m}$ & 13 \\
\hline Extensión & 15 a $100 \mathrm{~m}$ & 15 a $100 \mathrm{~m}$ & 15 a $40 \mathrm{~m}$ & 18 \\
\hline Proporción de Fachada/Superficie & o,3 a $1 \mathrm{~m} 2 / \mathrm{m} 2$ & 0,3 a $1 \mathrm{~m} 2 / \mathrm{m} 2$ & $1 \mathrm{~m} 2 / \mathrm{m} 2$ & $1 \mathrm{~m} 2 / \mathrm{m} 2$ \\
\hline Proporción de Ventanas/Superficie & o,06 a $1 \mathrm{~m} 2 / \mathrm{m} 2$ & 0,3 a $1 \mathrm{~m} 2 / \mathrm{m} 2$ & o,3 a $1 \mathrm{~m} 2 / \mathrm{m} 2$ & $0,3 \mathrm{~m} 2 / \mathrm{m} 2$ \\
\hline Proporción de Vtas. Soleadas/Superficie & o,015 a o,3 m2/m2 & o,3 m2/m2 & 0,08 a 0,3 m2/m2 & $0,3 \mathrm{~m} 2 / \mathrm{m} 2$ \\
\hline Consumo Energético & Bajo a Alto & Alto & Bajo & Bajo \\
\hline
\end{tabular}

consumo, y la fachada permite integrar ventanas colectoras, para el modelo de estudio se consideró un edificio de tamaño reducido y forma extendida, que permitiera una mayor superficie de fachada respecto a las plantas (Figura 1).

Por otro lado, en las fachadas se presentan desde un $20 \%$ al 100\% de ventanas (proporción entre superficie vidriada y opaca). Las proporciones más bajas, corresponden a edificios con ventanas pequeñas y con algunos costados opacos, y las proporciones más altas, a edificios con fachadas completamente vidriadas (denominados muros cortinas). De acuerdo a registros de edificios de oficinas en Santiago (26), los de menor consumo energético poseen relaciones de $24 \%$ a $44 \%$, debido a que mayores magnitudes vidriadas poseen mucha pérdida térmica nocturna y excesiva captación solar diurna o en verano, que requieren más calefacción y refrigeración. De modo que una proporción eficiente es en torno al 30\%, o sea de $0,3 \mathrm{~m} 2$ de ventana por cada metro cuadrado de fachada. Las ventanas están dispuestas usualmente en todas las fachadas, pero en latitudes medias (de los 40 a $30^{\circ}$ ) el costado soleado de un edificio recibe cerca de la mitad de la radiación solar que las cubiertas planas, mientras los laterales un cuarto, y el posterior un octavo. Por tanto en el modelo de estudio, se opta por concentrar la proporción vidriada en el costado soleado. Logrando de este modo una morfología que permite una superficie captadora en ventanas, cerca de cuatro veces superior a un edificio medio (equivalente a un edificio completamente vidriado), pero con mayor eficiencia (la mitad del consumo), al vanos hacia costados con baja captación solar.

Este modelo se puede considerar entonces como un caso particular de edificios de oficinas, pero es generalizable en cuan-

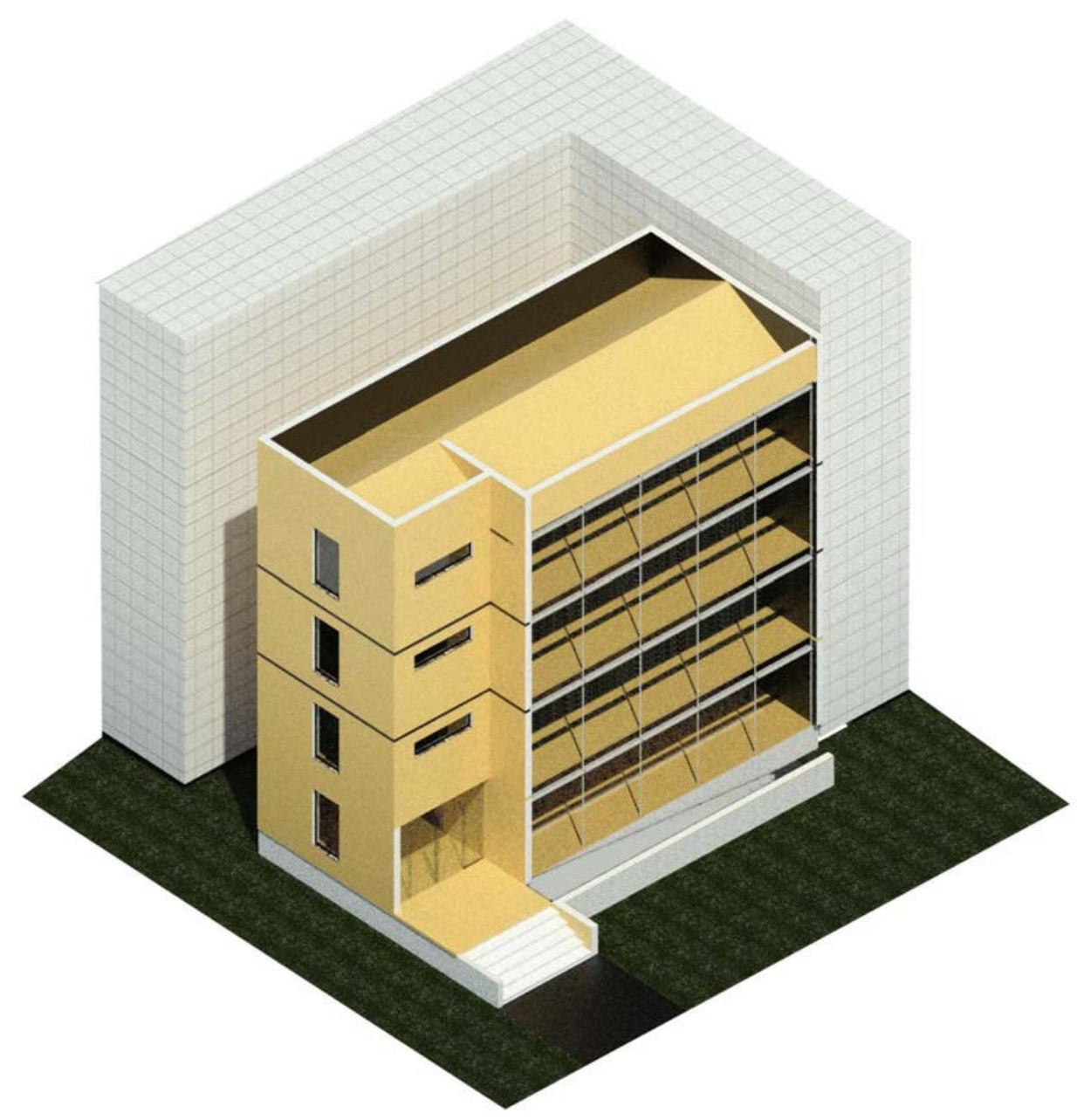

Figura 1. Modelo de estudio edificio de oficinas (elaboración propia). 
to éstas condiciones de forma y proporción vidriada se pueden regular en la planificación y diseño de nuevos edificios.

Las dimensiones del caso de estudio se consideraron entonces dentro del rango regular de edificación, pero de magnitudes reducidas para limitar su elaboración, y proporciones favorables para un menor consumo energético y mayor recolección solar, con una configuración usual en esta tipología. El modelo contempla una forma rectangular horizontal de 8 x $18 \mathrm{mts}$. de extensión (en sentido este-oeste), con cuatro plantas de 3 mts. de altura cada una, sobre un zócalo de un metro. En cada planta, un recinto menor de servicios, escalera y elevadores en un extremo, y la mayor parte abierta trabajo en escritorios (planta libre), totalizando $385 \mathrm{~m} 2$ de superficie ocupada. Con ventanas pequeñas en los extremos y en la fachada más soleada (norte) se consideró $120 \mathrm{~m} 2$ de ventanas continuas en las cuatro plantas. Considerando adosamiento en un extremo y un edificio próximo en el costado posterior.

\subsection{Simulaciones de Rendimiento y Generación Fotovoltaica}

Las simulaciones de comportamiento energético mediante software validados son una herramienta comprobada y normalizada para el estudio del rendimiento de edificios (30). Considerando la conformación de un modelo tridimensional, y una descripción de características materiales, equipamiento y ocupación, que entrega resultados de temperaturas por zona, flujos térmicos y requerimientos energéticos.

Los métodos y herramientas de cálculo de generación solar activa son más variados $(31,32)$. Los procedimientos difieren en antecedentes de radiación solar, rendimiento de los pa- neles y pérdidas de las instalaciones o entorno, etc. Además de plantear distintas estrategias de análisis, a partir de la demanda suplida o producción generada, o de la configuración de la instalación. También los procedimientos e instalaciones se han verificado para sistemas autónomos, pero escasamente para los integrados en la edificación. A pesar de estas limitaciones, en general los estudios suelen aproximarse en las magnitudes provistas y las mediciones han demostrado ser aproximadamente consistentes.

Para las simulaciones de rendimiento energético del caso de estudio se ocupó el software Design Builder, con el motor de cálculo Energy Plus, efectuando un modelo general del edificio (con datos generales que se indican en Tabla 3) y una secuencia de simulaciones modificando diferentes características. Considerando las cuatro plantas, cada una divida en dos zonas (oficinas y circulaciones). Primeramente con una conformación regular de la construcción y equipamiento, variando los horarios de ocupación para alcanzar una demanda equivalente a la media de consumos registrados para esta tipología en la zona, y así establecer una situación base de referencia. Posteriormente se probaron diferentes mejoramientos constructivos y equipos más eficientes, según las recomendaciones de edificación con menor consumo energético para esta zona (16). Luego se simularon modelos con diferentes localizaciones.

La base de datos de clima para realizar las simulaciones contempla: radiación solar global y difusa, porcentaje de cobertura de nubes, temperatura de bulbo seco, humedad relativa, velocidad y dirección del viento. Los datos deben ser en base horaria, ya que las simulaciones son de carácter dinámico. La base de datos de clima empleada es epw (Energy Plus weather files).

Tabla 3. Datos del modelo de simulación en caso base.

\begin{tabular}{|c|c|c|c|c|c|c|c|c|c|}
\hline Zona & un. & $\mathbf{1}$ & 2 & 3 & 4 & 5 & 6 & 7 & 8 \\
\hline Nivel & & 1 & 1 & 2 & 2 & 3 & 3 & 4 & 4 \\
\hline Destino & & Oficina & $\begin{array}{l}\text { Circulación y } \\
\text { Servicios }\end{array}$ & Oficina & $\begin{array}{l}\text { Circulación y } \\
\text { Servicios }\end{array}$ & Oficina & $\begin{array}{l}\text { Circulación } \\
\text { y Servicios }\end{array}$ & Oficina & $\begin{array}{l}\text { Circulación } \\
\text { y Servicios }\end{array}$ \\
\hline Superficie & $\mathrm{m} 2$ & 67,01 & 28,72 & 67,01 & 28,72 & 67,01 & 28,72 & 67,01 & 28,72 \\
\hline Ocupación & $\mathrm{p} / \mathrm{m} 2$ & 0,11 & 0,11 & 0,11 & 0,11 & 0,11 & 0,11 & 0,11 & 0,11 \\
\hline Calendario & & \multicolumn{8}{|c|}{ L-S 10:00 a 16:00 hrs } \\
\hline Actividad & & $\begin{array}{l}\text { Trabajo en } \\
\text { Escritorio }\end{array}$ & Pie/Caminar & $\begin{array}{l}\text { Trabajo en } \\
\text { Escritorio }\end{array}$ & Pie/Caminar & \begin{tabular}{|l|} 
Trabajo \\
en Escri- \\
torio
\end{tabular} & Pie/Caminar & $\begin{array}{l}\text { Trabajo en } \\
\text { Escritorio }\end{array}$ & Pie/Caminar \\
\hline Factor & & 0,8 & 0,9 & 0,8 & 0,9 & 0,8 & 0,9 & 0,8 & 0,9 \\
\hline Ropa/clo & Inv. & 1,79 & 1,79 & 1,79 & 1,79 & 1,79 & 1,79 & 1,79 & 1,79 \\
\hline & ver. & 0,61 & 0,61 & 0,61 & 0,61 & 0,61 & 0,61 & 0,61 & 0,61 \\
\hline DWH Consumo & $1 / \mathrm{m} 2-\mathrm{d}$ & 0,2 & o & 0,2 & 0 & 0,2 & 0 & 0,2 & 0 \\
\hline $\begin{array}{l}\text { Condiciones de } \\
\text { confort }{ }^{\circ} \mathrm{C}\end{array}$ & ${ }^{\circ} \mathrm{C}$ & $22^{\circ}$ a $26^{\circ}$ & $22^{\circ}$ a $26^{\circ}$ & $22^{\circ}$ a $26^{\circ}$ & $22^{\circ}$ a $26^{\circ}$ & $22^{\circ}$ a $26^{\circ}$ & $22^{\circ}$ a $26^{\circ}$ & $22^{\circ}$ a $26^{\circ}$ & $22^{\circ}$ a $26^{\circ}$ \\
\hline $\begin{array}{l}\text { Mínimo de aire } \\
\text { fresco }\end{array}$ & $1 / \mathrm{s}$ & 10 & 10 & 10 & 10 & 10 & 10 & 10 & 10 \\
\hline $\begin{array}{l}\text { Densidad de } \\
\text { iluminación }\end{array}$ & $\mathrm{W} / \mathrm{m} 2$ & 8 & 8 & 8 & 8 & 8 & 8 & 8 & 8 \\
\hline $\begin{array}{l}\text { Ganancias por } \\
\text { equipos }\end{array}$ & $\mathrm{W} / \mathrm{m} 2$ & 4,5 & 4,5 & 4,5 & 4,5 & 4,5 & 4,5 & 4,5 & 4,5 \\
\hline Muros exteriores & $\mathrm{U}$ & 1,6 & 1,6 & 1,6 & 1,6 & 1,6 & 1,6 & 1,6 & 1,6 \\
\hline Cubierta & $\mathrm{U}$ & 1,6 & 1,6 & 1,6 & 1,6 & 1,6 & 1,6 & 1,6 & 1,6 \\
\hline Suelos & $\mathrm{U}$ & 0,69 & 0,69 & 0,69 & 0,69 & 0,69 & 0,69 & 0,69 & 0,69 \\
\hline Ventanas & $\mathrm{U}$ & 2,55 & 2,55 & 2,55 & 2,55 & 2,55 & 2,55 & 2,55 & 2,55 \\
\hline Infiltraciones & $\mathrm{ac} / \mathrm{h}$ & 0,6 & 0,6 & 0,6 & 0,6 & 0,6 & 0,6 & 0,6 & 0,6 \\
\hline HVAC & & $\begin{array}{l}\text { VRF (Air- } \\
\text { Cooled), } \\
\text { HR-DOAS }\end{array}$ & \begin{tabular}{|l} 
VRF \\
(Air-Cooled), \\
HR DOAS
\end{tabular} & \begin{tabular}{|l} 
VRF (Air- \\
Cooled), \\
HR DOAS
\end{tabular} & \begin{tabular}{|l|} 
VRF \\
(Air-Cooled), \\
HR DOAS \\
\end{tabular} & $\begin{array}{l}\text { VRF (Air- } \\
\text { Cooled), } \\
\text { HR DOAS }\end{array}$ & \begin{tabular}{|l} 
VRF (Air- \\
Cooled), \\
HR DOAS
\end{tabular} & $\begin{array}{l}\text { VRF (Air- } \\
\text { Cooled), } \\
\text { HR DOAS }\end{array}$ & $\begin{array}{l}\text { VRF } \\
\text { (Air-Cooled), } \\
\text { HR DOAS }\end{array}$ \\
\hline
\end{tabular}


Para el cálculo de generación energética se utilizó el software PVsyst, que integra el aporte con la demanda horaria, considerando la superficie disponible en la fachada más soleada del caso de estudio, así como las condiciones climatológicas de Santiago de Chile. Las ventanas están compuestas por un paramento continuo con vidrios sobrepuestos inclinados que contienen la capa luminiscente, que actualmente puede ser parcialmente opaca y sin coloración, contribuyendo a reducir el sobrecalentamiento y deslumbramiento), así como absorber y retransmitir parte de la radiación incidente hacia el perímetro con células fotovoltaicas para convertirla en energía eléctrica. La eficiencia de conversión se define como el cociente entre la potencia generada y la irradiancia incidente sobre todo el vidrio colector, en condiciones estándar de medida (1000 W/m2 de irradiancia, $25^{\circ} \mathrm{C}$ de temperatura de ambiente y espectro AM1.5). La eficiencia de conversión se ve comprometida para que la mayor parte de la radiación solar incidente atraviese los vidrios, para mantener iluminación natural y asegurar un consumo regular de alumbrado eléctrico.

Varios grupos de investigación han publicado eficiencias de conversión de concentradores luminiscentes que oscilan entre el $2,7 \%$ y el $7,1 \%(33,34,35)$. Estos sistemas utilizan en la mayoría de los casos reflectores posteriores que consiguen aumentar considerablemente la eficiencia del colector pero que impiden su utilización como ventana al bloquear toda la luz incidente. Al ser trabajos de investigación, el área de los colectores es muy pequeña, en general del orden de $5 \times 5 \mathrm{~cm}$. En el proyecto WINNER en de- sarrollo por los autores se consideran fragmentos de $15 \mathrm{x} 1$ $5 \mathrm{~cm}$. Las eficiencias estimadas oscilan entre el $1 \%$ y el $4 \%$, en función del porcentaje de luz que se permita bloquear, la eficiencia del concentrador y la del sistema fotovoltaico ópticamente acoplado al mismo.

Basándose en este análisis, se realizaron varias simulaciones definiendo en el software dos nuevos tipos de fotovoltaicos con eficiencias $1 \%$ y $4 \%$ y características basadas en células de silicio multicristalino. Contando con un área disponible de $120 \mathrm{~m} 2$ en la fachada norte del edificio, y asumiendo varias inclinaciones. Se escogió en cada caso el inversor más apropiado para la instalación, y se despreciaron posibles sombras de edificios colindantes. Para los valores de pérdidas por diferentes condiciones de temperatura, irradiancia, así como eficiencia de la conversión DC-AC y pérdidas en el cableado, se consideraron valores típicos dados en el software PVSyst. Una característica importante de las ventanas solares basadas en LSC es que, a diferencia de otros tipos de concentradores, no requieren de seguimiento pues son capaces de concentrar tanto la radiación directa como la difusa.

\section{RESULTADOS}

\subsection{Demanda}

Los resultados de simulaciones del modelo base del edificio de oficinas presentaron un comportamiento convencional de

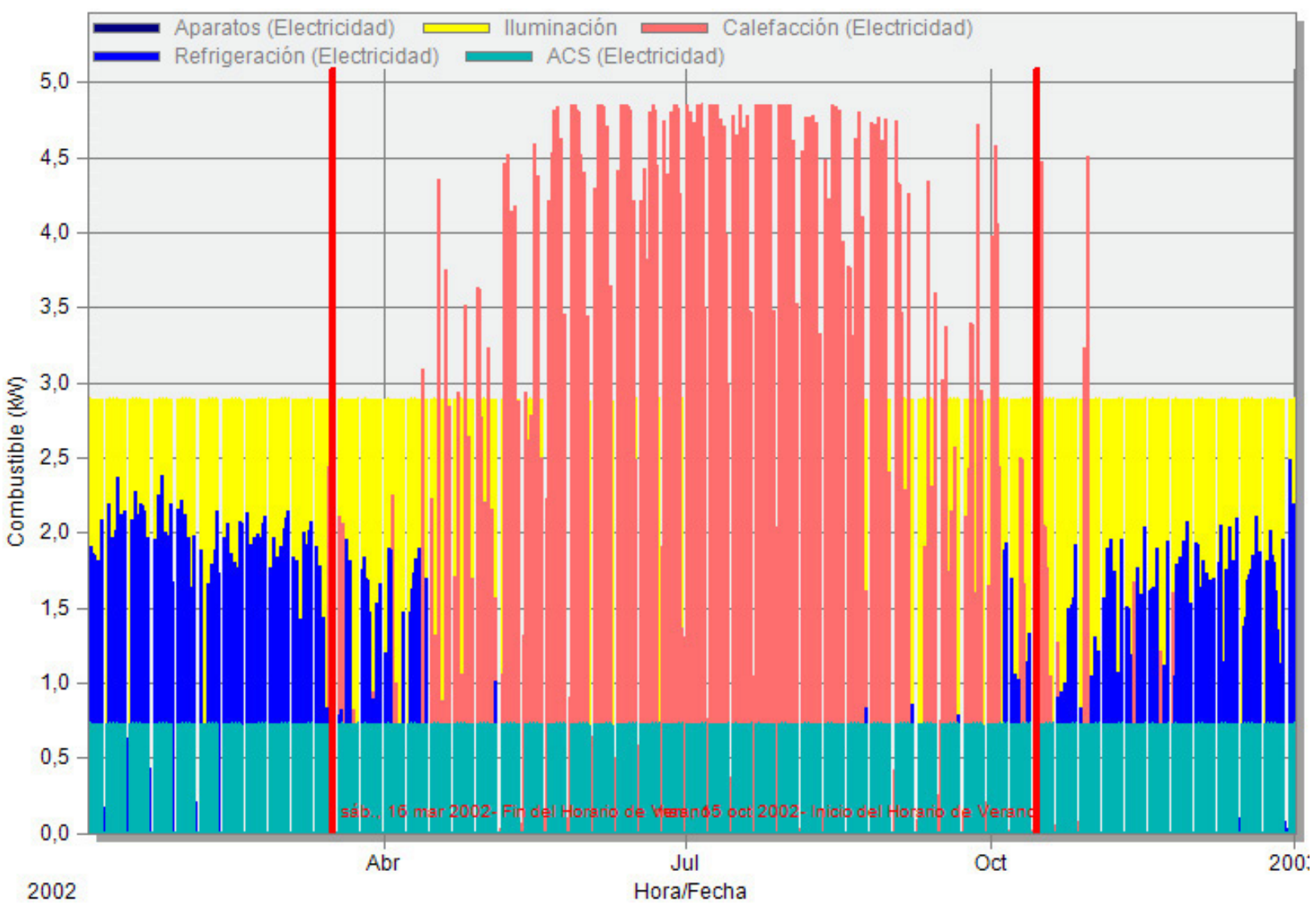

Figura 2. Gráfico de demandas horarias por servicio en caso base (elaboración propia). 


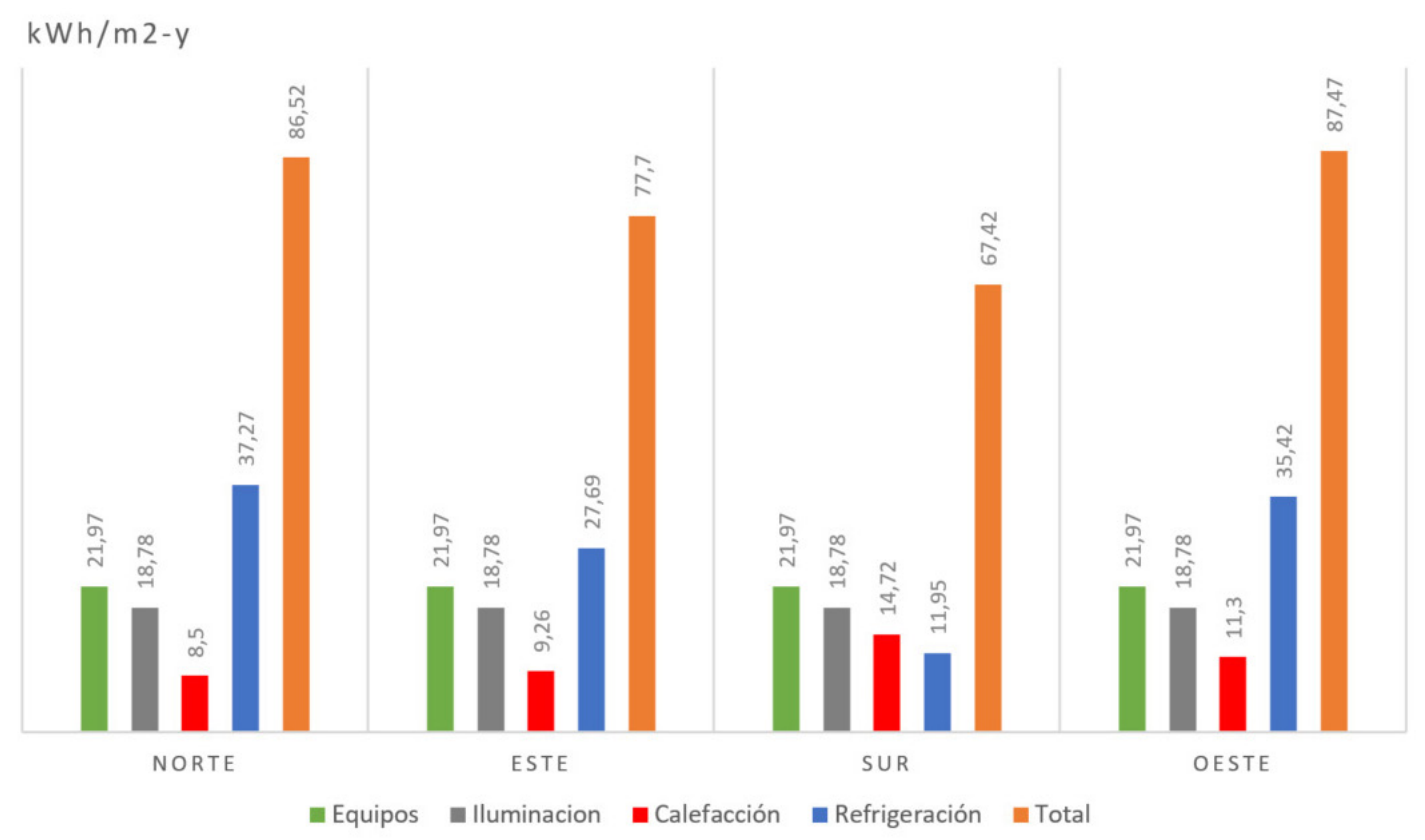

Figura 3. Gráfico de demandas anuales por servicio y totales en caso base con distintas orientaciones solares (elaboración propia).

demandas (Figura 2). Una fracción relevante de los requerimientos energéticos se origina por los equipos de trabajo, con bastante estabilidad anual según horarios de ocupación, y otra fracción regular se origina por iluminación que también es bastante estable. Una parte equivalente esta originada por climatización, tanto de calefacción como de refrigeración, de manera estacional invertida. La calefacción se incrementa en periodos invernales y la refrigeración en verano. Considerando variaciones de ocupación se ajustaron los resultados por superficie, determinando una utilización de lunes a sábado de 10 AM a 4 PM, o sea 30 horas semanales (con una densidad de $0,1 \mathrm{p} / \mathrm{m} 2$ ), se obtuvo una demanda de $86,5 \mathrm{kWh} / \mathrm{m} 2-\mathrm{y}$ equivalente a los estudios para esta tipología en la zona (27, $28,29)$. Este horario es usual, considerando los contratos de trabajo son por 44 hrs. incluyendo actividades en terreno, y los horarios hábiles se suelen extender en periodos centrales del día.

Las simulaciones del modelo con distintas orientaciones solares, arrojaron totales diversos, debido a diferencias en climatización, ya que las demandas de equipamiento e iluminación se mantuvieron casi constantes (Figura 3). El modelo con ventanal al costado sur, menos soleado, presentó la menor demanda total $(67,42 \mathrm{kWh} / \mathrm{m} 2-\mathrm{y})$, debido a un requerimiento de refrigeración más bajo. Mientras el modelo con ventanal al este entrego una demanda intermedia $(77,7 \mathrm{kWh} / \mathrm{m} 2-y)$ y al oeste, un poco más alta $(87,42 \mathrm{kWh} /$ $\mathrm{m} 2-\mathrm{y})$, en ambos casos con variaciones de refrigeración,

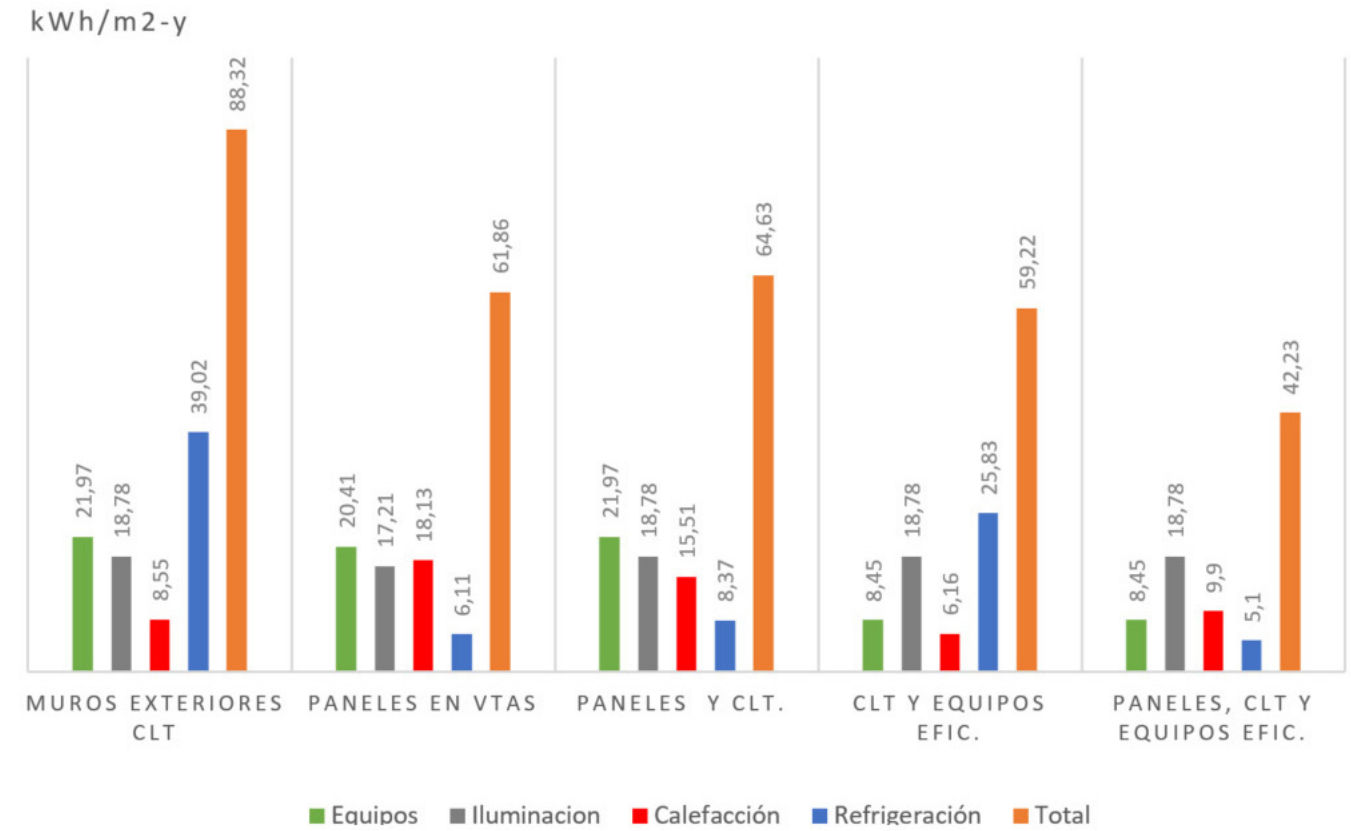

Figura 4. Gráfico de demandas anuales por servicio y total en casos mejorados (elaboración propia). 
algo menores en calefacción, reflejando la incidencia del sobrecalentamiento de verano, y en particular del atardecer, por la concentración de superficie vidriada (Figura 4). Como también el comportamiento cambiante evidencia el carácter dinámico de las estrategias constructivas.

Los modelos con mejoramientos constructivos y de equipamiento presentaron variaciones relevantes, exceptuando el cambio de muros por placas de madera CLT que arrojó una demanda levemente superior al modelo base $(88,32 \mathrm{kWh} /$ m2-y). Aunque esta alternativa constructiva incrementa la resistencia térmica y hermeticidad de la envolvente vertical, este resultado refleja nuevamente el carácter variable del rendimiento térmico de las edificaciones. Se reducen las demandas de refrigeración por menores perdidas de la envolvente, pero aumentan de calefacción, probablemente por la menor masa térmica. Sin embargo, en el modelo con paneles en las ventanas se presenta una reducción importante de la demanda global (61,86 kWh/m2-y), con una bajada importante de la refrigeración, aparentemente por el sombreamiento de las placas que reduce el sobrecalentamiento interior, aunque aumenta la calefacción por las menores ganancias térmicas. El modelo combinado de paneles en ventanas con muros de placas CLT, entrega un comportamiento levemente superior $(64,63 \mathrm{kWh} / \mathrm{m} 2-y)$, en que se reduce calefacción, pero aumenta refrigeración. La simulación del modelo con placas CLT y equipos eficientes arroja resultados similares $(59,22$ $\mathrm{kWh} / \mathrm{m} 2-\mathrm{y})$, aunque con un incremento de refrigeración. Mientras el modelo con placas CLT, paneles en ventanas y equipos eficientes logra una reducción relevante $(42,33 \mathrm{kWh} /$ m2-y), evidenciando que el remplazo de los equipos tradicionales de trabajo e iluminación por productos de mejor rendimiento posee una contribución importante en la minimización de los consumos.

\subsection{Aporte Energético}

La producción energética de las ventanas en el modelo ubicado en Santiago de Chile, calculada mediante el software PVSyst, con paneles de eficiencias de $1 \%$ y $4 \%$, y con varios ángulos de inclinación, se muestra en la Tabla 4.

Esta producción corresponde de un 5,6\% a un 41,6\% de la demanda total $(16.258,55 \mathrm{kWh})$, o también de un $12,7 \%$ a un $93,7 \%$ de los requerimientos de iluminación $(7.230,3$ $\mathrm{kWh}$ ). Por tanto, sin requerir acumulación y otorgando una provisión relevante para la ocupación regular del edificio.

Respecto a los rendimientos según el ángulo de inclinación del vidrio recolector respecto a la horizontal, son similares con equipos de diferentes eficiencias. En los $30^{\circ}$ se produce la mayor cantidad de energia producida, decreciendo sustancialmente hacia la vertical. Este ángulo se aproxima a la latitud $\left(33^{\circ}\right)$, lo que es consistente con las recomendaciones técnicas y permite un adecuado afianzamiento, sombreamiento y visión del entorno. La posición horizontal $\left(\mathrm{O}^{\circ}\right)$ es mas compleja de sustentar y genera mayor sombreamiento.

\subsection{Balance de Energía}

De acuerdo a las simulaciones realizadas, en el gráfico de balance de cero-energía (12), se puede considerar que el edificio en condiciones regulares presentaría un demanda de 86,52 $\mathrm{kWh} / \mathrm{m} 2-\mathrm{y}$ sin aportes. Con características constructivas mejoradas alcanzaría 59,22 kWh/m2-y y de equipamiento eficiente 42,33 kWh/m2-y. El aporte total de las ventanas con LSC podría alcanzar 17,61 kWh/m2-y para la superficie ocupada, logrando suplir hasta un 41,6\% del edificio mejorado (Figura 5). Sin embargo para un edificio solo de condiciones constructivas mejoradas, este aporte representa menos de un tercio $(29,7 \%)$ y para un edificio usual, cerca de un quinto $(20,35 \%)$. Con la fachada en otras orientaciones, la generación puede ser menos de la mitad, hasta un octavo de la demanda. Por lo que en condiciones específicas de diseño, construcción y equipamiento, las ventanas con LSC en extensiones amplias pueden aportar hasta cerca de la mitad de la energía requerida para un edificio, lo que constituye un paso relevante para un balance cero-energía.

Una posibilidad adicional de aporte es la instalación de paneles colectores fotovoltaicos en techumbre, que de acuerdo a la superficie de cubierta disponible en este edificio (100 m2) y su localización (Lat. $33^{\circ}$ ), con una inclinación y orientación optima $\left(33^{\circ}-\mathrm{O}^{\circ}\right)$ y eficiencia regular $(11,98 \%)$, según cálculos estáticos podría alcanzar un total anual de 10.197 kWh-y. Esto representaría 26,48 kWh-y/m2, es decir superior al aporte de las ventanas con LSC (por la mayor eficiencia) y más de la mitad de la demanda en el edificio acondicionado, que complementado con la recolección de ventanas permitiría aportar el total de la energía requerida, alcanzando un balance cero. Sin embargo se debe considerar que estos aportes suelen ser sincrónicos, por tanto deben analizarse conjuntamente las perdidas por baterías o contribuciones a la red.

\subsection{Variaciones}

En relación al balance cero-energía, podemos advertir en la morfología del edificio, que para aumentar la recolección, requiere incrementar la superficie de fachada (mediante una mayor altura entre pisos o la cantidad de pisos, lo que implica mayor superficie o espacio de demanda), o reducir el área ocupada (estrechando el volumen, que es poco funcional). También una mayor extensión horizontal para captación en cubierta, produce mayor superficie de demanda,

Tabla 4. Resultados de la simulación de producción energética con ventanas en superficie de 120 m2 con orientación norte.

\begin{tabular}{|c|c|c|c|c|}
\hline $\begin{array}{c}\text { Eficiencia de } \\
\text { ventana solar }\end{array}$ & Inclinación & $\begin{array}{c}\text { Irradiación anual sobre plano } \\
\text { receptor (kWh) }\end{array}$ & Factor de rendimiento & $\begin{array}{c}\text { Producción anual } \\
\text { (kWh) }\end{array}$ \\
\hline $1 \%$ & $90^{\circ}$ & 1055 & 0,76 & 923 \\
\hline $1 \%$ & $60^{\circ}$ & 1625 & 0,76 & 1442 \\
\hline $1 \%$ & $30^{\circ}$ & 1881 & 0,76 & 1650 \\
\hline $4 \%$ & $90^{\circ}$ & 1055 & 0,78 & 3861 \\
\hline $4 \%$ & $60^{\circ}$ & 1625 & 0,78 & 5932 \\
\hline $4 \%$ & $30^{\circ}$ & 1881 & 0,78 & 6778 \\
\hline
\end{tabular}




\section{Generación $\mathrm{kWh} / \mathrm{m} 2$}

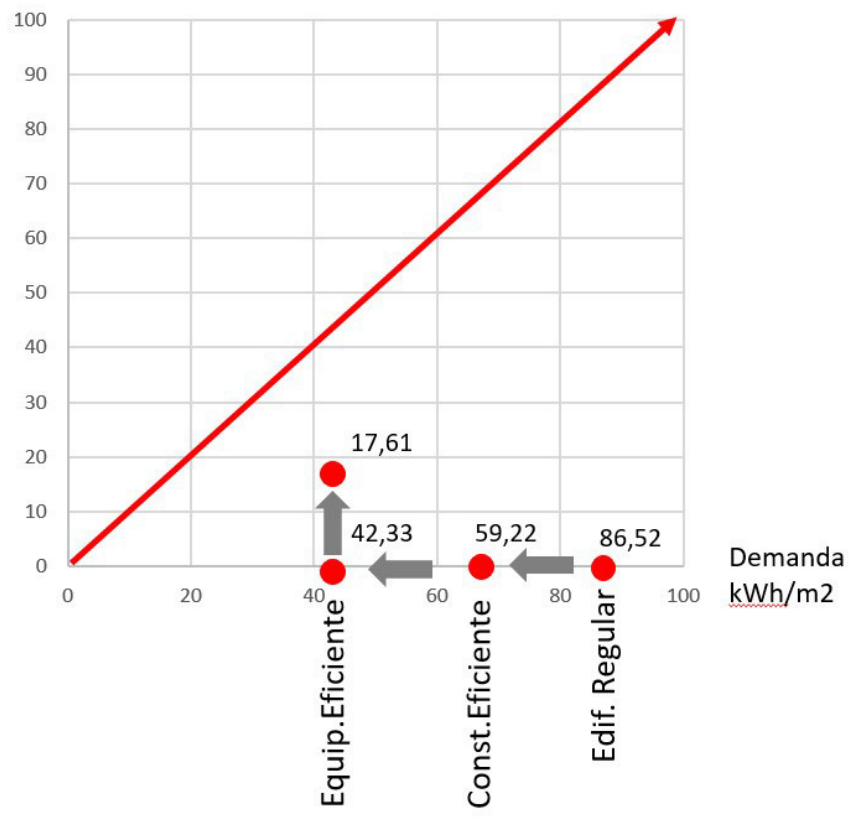

Figura 5. Gráfico de balance energético (elaboración propia).

y por ende menor proporción del aporte, especialmente de ventanas que mantienen su magnitud o se reducen. Otras relaciones horizontales, reducen la orientación norte y la radiación recibida y energía recolectada. Por tanto, aumentar dimensiones del volumen del edificio pueden alejarlo de la meta de cero-energía. A su vez las características constructivas adoptadas demostraron en las simulaciones una incidencia limitada. Por tanto acciones mayores (por ejemplo más aislación térmica), son más costosas y probablemente tengan una influencia más reducida, y por ende poco efectivas. La implementación de equipos de iluminación y climatización más eficiente, demostraron una reducción significativa, pero otras alternativas no son factibles de implementar (como más iluminación natural) o poco efectivas (como la geotermia o generadores eólicos), de acuerdo a experi- mentaciones realizadas. De modo, que en estas condiciones morfológicas, constructivas y de equipamiento, el modelo se encuentra cerca de las características límite para la meta de cero energía.

La localización del edificio presenta variaciones relevantes. La simulación del edificio mejorado en una latitud más baja, cercana al Ecuador, según el archivo climático de la ciudad de Antofagasta, en lat. $23^{\circ}$, reduce la demanda a 34,94 kWh/m2-y, debido a poseer temperaturas más altas, y por ende menores requerimientos de calefacción (aunque se incrementa la refrigeración). Mientras que la simulación en una latitud más alta, según el archivo climático de la ciudad de Punta Arenas, en Lat. $41^{\circ}$, con temperaturas estacionales más bajas, incrementa la demanda a 71,53 kWh/m2-y.

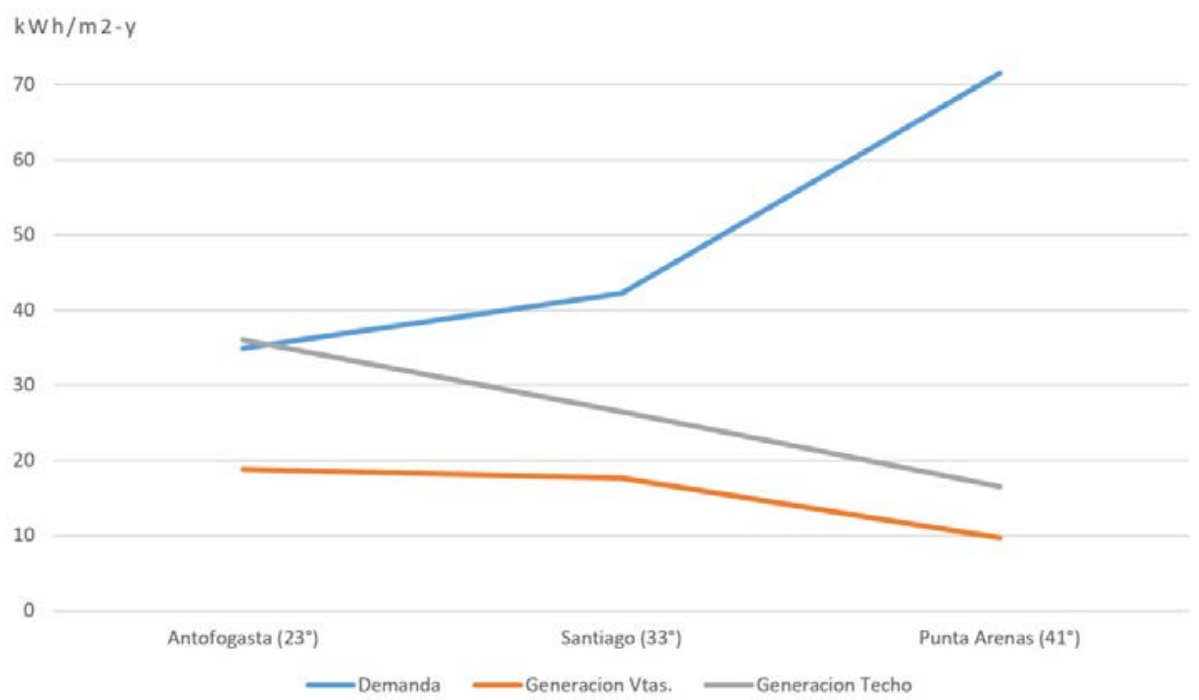

Figura 6. Gráfico de demandas y aportes en distintas localizaciones (elaboración propia). 
A su vez, el aporte de cubierta, se incrementa con latitudes más bajas con radiación más alta, alcanzando 34,94 kWh/ m2-y en Antofagasta; mientras en Punta Arenas, se reduce a $16,8 \mathrm{kWh} / \mathrm{m} 2-y$ por la menor radiación. La contribución de ventanas, presenta un leve aumento en Antofagasta obteniendo 18,86 kWh/m2-y, probablemente debido a que en el primer caso aumenta la radiación, pero también el ángulo de inclinación, y en Punta Arenas se reduce a 9,74 kWh/ m2-y. Evidenciando un comportamiento más estable en latitudes medias-bajas. Considerando el aporte conjunto, y la reducción de demanda, el balance se aproxima a la meta cero energía, al reducirse la latitud (Figura 6). Aunque es probable que en latitudes más bajas aumenten la demanda por refrigeración.

La ocupación del edificio presenta también una variación relevante en el comportamiento energético. Considerando 20 horas de ocupación semanales (unas pocas horas diarias), la demanda alcanza los $28 \mathrm{kWh} / \mathrm{m} 2-y$, o sea próximo al balance cero-energía con aporte de ventanas. La ocupación con 77 horas semanales, alcanza los $80 \mathrm{kWh} / \mathrm{m} 2-\mathrm{y}$, alejándose de la meta cero-energía. Esta oscilación puede afectar el destino funcional del edificio, y solo permite inferir que la mayor ocupación dificulta alcanzar las metas, y puede considerarse una distribución de funciones entre edificios o agrupar recintos con alta y baja ocupación. De modo que en términos generales, el modelo estudiado presenta condiciones favorables para alcanzar metas cero-energía con ventanas fotovoltaicas con condensadores, complementadas con paneles en cubierta. Edificios con distintas características presentan aportes menores, sin embargo la localización presenta posibilidades amplias e incluso en latitudes más bajas y climas más cálidos, algo más favorables, lo que admitiría edificios más variados.

\section{CONCLUSIONES}

Este trabajo evalúa la integración de ventanas fotovoltaicas con LSC para edificios cero-energía. Revisando primeramente planes estatales de Europa y Estados Unidos, que promueven un balance casi nulo de energía en la operación de edificios, a través de reducir consumos e integrar renovables. Aunque las métricas y condiciones no se encuentran cabalmente consensuadas, y en Chile se presentan metas más reducidas y orientadas a la gestión de energía, las diferentes perspectivas son concurrentes hacia disminuir requerimientos e integrar aportes energéticos. Por lo que ventanas fotovoltaicas con LSC pueden contribuir en esta tendencia.
Luego se revisaron condiciones de edificios de oficinas en Santiago de Chile, que presentan una configuración y rendimiento energético variable por razones climáticas y constructivas. Se elaboró un modelo de simulación, con morfología apropiada para instalar ventanas en una mayor relación de superficie respecto al área de demanda, con un rendimiento energético similar a la media de esta tipología en la zona. Con medidas de eficiencia constructiva y de equipamientos se determinó una reducción del consumo, y un aporte de ventanas fotovoltaicas con LSC, en diferentes ángulos y eficiencias, alcanzando hasta un $40 \%$ del consumo. Considerando un desarrollo de la tecnología e integración constructiva, a costes rentables. Lo cual se puede complementar con generación en cubierta mediante paneles fotovoltaicos regulares, logrando un balance nulo. Aunque puede tener pérdidas horarias. Además con condiciones morfológicas y técnicas que deben ser verificadas. En edificios con otras características el aporte probablemente será menor. Sin embargo, las características del edificio, como de los productos considerados, se encuentran dentro de las tendencias de desarrollo y planes nacionales sobre energía en la edificación. La variación de condiciones presenta una convergencia hacia las características determinadas, incluyendo una estabilidad por localización.

Por tanto, en general se sugiere para que las ventanas fotovoltaicas de vidrios con LSC puedan contribuir en lograr edificios cero-energía en climas templados de latitudes medias, deberían aplicarse en amplias extensiones continuas sobre los costados soleados. Manteniendo una proporción extendida del volumen (en sentido Este-Oeste), para otorgar una mayor relación de aporte respecto al consumo, y sus restantes fachadas opacas para asegurar un desempeño térmico con reducido consumo energético. Considerando también paramentos con baja transmitancia, buena hermeticidad y masa térmica, equipamiento de alta eficiencia y protección solar (que puede ser provista por la opacidad de los vidrios), e integración de otras fuentes renovables. De modo que la tecnología LSC puede aportar para alcanzar un balance energético casi nulo en condiciones específicas y contribuir en la generalidad de los edificios a un rendimiento más sostenible.

\section{AGRADECIMIENTOS}

Se agradece al Proyecto WINNER ELAC2014/ESE0146, al arquitecto Alberto Nope y Helena Aguilar, de Nanolayer Coating Technologies Ltda.

\section{REFERENCIAS}

(1) Tsoi, S. (2012) Structured luminescent solar energy concentrators: a new route towards inexpensive photovoltaic energy, Doctoral Thesis, Eindhoven:Tecnische Universitet Eindhoven.

(2) Kerrouche A., D. A. Hardy, D. Ross, B. S. Richards (2014) Luminescent solar concentrators: From experimental validation of 3D ray-tracing simulations to coloured stained-glass windows for BIPV, Solar Energy Materials and Solar Cells, 122, 99-106

(3) Aste N., Tagliabue L. C., Del Pero C., Testa D., Fusco R. (2015); Performance analysis of a large-area luminescent solar concentrator module, Renewable Energy, 76, 330-337.

(4) Hardy, D.A., Blekastad, S., Kerrouche, A., Roaf, S.C. and Richards, B.S. (2012) Creative use of BIPV materials: barriers and solutions. 27th European Photovoltaic Solar Energy Conference and Exhibition, Messe Frankfurt, Frankfurt, Germany. 
(5) Torcellini, P.; Pless, S.; Deru, M. y Crawley, D. (2006). Zero Energy Buildings: A Critical Look at the Definition. Golden:National Renewable Energy Laboratory.

(6) National Institute of Buildings Sciences (2015) A Common Definition for Zero Energy Buildings. Washington:US Department of Energy.

(7) EP-CEU (2010); Directive 2010/31 on the energy performance of buildings. The European Parliament and the Council of the European Union.

(8) Hermelink A.; Schimschar S.; Boermans T.; Pagliano L; Zangheri P.; Armani R.; Voss, K.; Musall E.; (2012) Towards nearly zero energy buildings Definition of common principles under the EPB, Ecofys, Koln.

(9) Kurnitski J.; Allard F.; Braham D.; Goeders G.; Heiselberg P.; Jagemar L.; Kosonen R.; Lebrun J.; Mazzarella L.; Railio J.; Seppänen O.; Schmidt M.; Virta M.; (2011) How to define nearly net zero energy buildings nZEB- REHVA proposal for uniformed national implementation of EPBD recast, REHVA-Journal, 6-12.

(10) Sartori I.; Napolitano A.; Marszal A. J.; Pless S.; Torcellini P. and Voss K. Criteria for Definition of Net Zero Energy Buildings, EuroSun Conference Graz 2010.

(11) Ayman M.; Ala H.; Kai S.; (2014) Fulfilment of net-zero energy building (NZEB) with four metrics in a single family house with different heating alternatives. Applied Energy, 114, 385.

(12) Deng S., Wang R. Z., Daa Y. J. (2014), How to evaluate performance of net zero energy building, a literature research, Energy 71 (2014) 1-16

(13) Scognamiglioa A., Adinolfia G., Graditia G., Saretta E. (2014); Photovoltaics in Net Zero Energy Buildings and Clusters: enabling the smart city operation, Energy Procedia, 61, 1171 - 1174

(14) Ares E. (2016), Zero Carbon Homes, Londres:House of Commons Library.

(15) Crawley, D. Pless S y Torcellini, P. (2009). Getting to Net Zero Energy Buildings. National Renewable Laboratory.

(16) Instituto de la Construcción (2012) Manual de Gestión de Energía en Edificios Públicos, Santiago: Innova-Corfo.

(17) MINVU (2013). Estrategia Nacional de Construcción Sustentable 2013-2020, Ministerio de Vivienda y Urbanismo (MINVU), Chile.

(18) MMA (2014). Planes de Descontaminación Atmosférica Estrategia 2014 - 2018, Ministerio del Medio Ambiente (MMA), Chile.

(19) MINER (2015). Política Energética de Chile 2050, Ministerio de Energía, Chile.

(20) CORFO (2016). Hoja de Ruta CONSTRUYE 2025, Corporación de Fomento, Chile.

(21) Strong S. (2011). Building Integrated Photovoltaics (BIPV). [en línea]. Disponible en: https://www.wbdg.org/resources/bipv.php.

(22) Voss, K. y Musall, E. (2012). Net Zero Energy Buildings: International projects of carbon neutrality in buildings. Karlsruhe:ENob.

(23) Kim J-H; Kim H-R y Kim J-T (2015); Analysis of Photovoltaic Applications in Zero Energy Building Cases of IEA SHC/ EBC Task 40/Annex 52, Sustainability, 7, 8782-8800; doi:10.3390/su7078782

(24) Gallo A., Téllez Molina B., Prodanovic M., González Aguilar J., Romero M. (2014); Analysis of net Zero-Energy Building in Spain. Integration of PV, solar domestic hot water and air-conditioning systems, Energy Procedia 48, 828 - 836

(25) Aste N., Tagliabue, L. C., Palladino P., Testa D. (2015), Integration of a luminescent solar concentrator: Effects on daylight, correlated color temperature, illuminance level and color rendering index, Solar Energy, 114, 174-182.

(26) Vasquez, C.; Encinas, F. y D’Alencon, R. (2015). Edificios de oficinas en Santiago: ¿Qué estamos haciendo desde el punto de vista del consumo energético? $A R Q, 89,50-61$, http://dx.doi.org/10.4067/So717-69962015000100008

(27) Pareja, P. A., (2007). Desarrollo de índices de desempeño del consumo energético y de agua sanitaria en edificios de oficinas de Santiago. Tesis, Santiago: Universidad de Chile.

(28) Bustamante W., De Herde, A., y Encinas, F. (2011), Análisis de comportamiento térmico de edificios de oficinas en comunas de la Región Metropolitana, Chile. Revista de la Construcción. 10:1, 64-77

(29) Price Waterhouse Coopers (2007) Desarrollo de una línea base y metodología de medición del consumo energético en edificios, Santiago: Corfo.

(30) Garcia-Alvarado, R., Gonzalez, A., Bustamante, W., Bobadilla, A. y Muñoz, C., (2014). Características relevantes de la simulación energética de viviendas unifamiliares. Informes de la Construcción, 66- 533, DOI 10.3989/ic.12.108.

(31) Frontini F., Marzoli M., Doust N. (2013), Investigation of different simulation tools for solar photovoltaic modules, IBS$P A-2013,89-94$

(32) Axaopoulos, P. J., Fylladitakis, E. D. \& Gkarakis, K. (2014) Accuracy analysis of software for the estimation and planning of photovoltaic installations, Int J Energy Environ Eng 5:71

(33) Currie M. J. et al. (2008), High-efficiency Organic Solar Concentrators for Photovoltaics, Science 321, 226.

(34) Sloof L. H. (2008), A luminescent solar concentrator with 7.1\% power conversion efficiency, phys. stat. sol. (RRL) 2, No. 6, 257-259.

(35) Goldschmidt J. C. et al. (2009), Increasing the efficiency of fluorescent concentrator systems, Solar Energy Materials \& Solar Cells 93, 176-182. 